HRJ

v.2 n.11 (2021)

Recebido: $13 / 11 / 2020$

Aceito: $27 / 04 / 2021$

\title{
Abordagem de via aérea difícil em paciente com Síndrome Orofaciodigital tipo II ou Síndrome de Mohr: um relato de caso
}

\author{
Pedro Thiago Hideyuki Takagi ${ }^{1}$ \\ Bruno Zeuxis de Siqueira ${ }^{1}$ \\ Guilherme Peixoto Mendonça ${ }^{1}$ \\ Adriana Navarro Machado ${ }^{2}$ \\ André Santana Prata ${ }^{3}$
}

\begin{abstract}
${ }^{1}$ Médico residente em Anestesiologia no Hospital Regional de Taguatinga - HRT
${ }^{2}$ Médica anestesiologista preceptora do programa de residência médica de Anestesiologia do Hospital Regional de Taguatinga - HRT

${ }^{3}$ Médico anestesiologista chefe do programa de residência médica de Anestesiologia do hospital Regional de Taguatinga - HRT
\end{abstract}

\section{RESUMO}

A síndrome orofaciodigital é um transtorno raro do desenvolvimento embrionário. Os pacientes podem apresentar alterações de boca, face, mãos e pés. Há descrito pelo menos 11 formas clínicas diferentes da síndrome, entre elas a síndrome orofaciodigital tipo II ou síndrome de Mohr. A abordagem de via aérea na síndrome orofaciodigital pode ser um desafio, já que as alterações craniofaciais como lábio leporino/fenda palatina, presentes na síndrome, são preditores de via aérea difícil em pediatria. O relato de caso descreve uma lactente de 4 meses, portadora de Síndrome de Mohr que foi admitida com sintomas respiratórios e, durante internação, apresentou parada cardiorrespiratória, sendo realizada laringoscopia direta por diversas vezes, sem sucesso na intubação orotraqueal. As diferenças anatômicas explicam o potencial maior de dificuldades técnicas das vias aéreas em lactentes do que em adolescentes ou adultos. Em geral, as vias aéreas dos lactentes diferem das de adultos por cinco pontos: 1) tamanho relativamente grande da língua da criança em relação à orofaringe; 2) a laringe tem localização mais cefálica no pescoço; 3) a epiglote é curta, em forma de ômega e angulada sobre a entrada da laringe; 4) as cordas vocais são anguladas, consequentemente, um tubo traqueal que passou cegamente pode facilmente se alojar na comissura anterior em vez de deslizar para dentro da traqueia; 5) a laringe é em forma de funil, sendo a porção mais estreita localizada na cartilagem cricoide. Os guidelines de via aérea difícil em pediatria são ferramentas essenciais, nesse contexto, para prevenção de complicações associadas sendo assim imprescindível o domínio destes por profissionais intensivistas e anestesiologistas. O compartilhamento dessas informações 
se torna importante já que há poucos dados na literatura sobre abordagem de via aérea em paciente com Síndrome de Mohr.

Palavras-chave: Síndrome orofaciodigital tipo II; Síndrome Mohr; Manejo de via aérea; Via aérea difícil

\title{
Difficult airway approach in patient with Oro-Facial-Digital syndrome type II or Mohr syndrome: a case report
}

\begin{abstract}
Oro-facio-digital syndrome is a rare disorder of embryonic development. Patients may have deformities in the mouth, face, hands and feet. At least 11 different clinical forms of the syndrome have been described, including oro-facial-digital syndrome type II or Mohr syndrome. The approach of the airway in the oro-facial-digital syndrome can be a challenge because craniofacial changes such as leborine lip / cleft palate, present in the syndrome, are predictors of difficult airway in pediatrics. The case report describes a 4month-old infant with Mohr Syndrome who was admitted with respiratory symptoms and during hospitalization presented cardiac arrest, with direct laryngoscopy performed several times, without success in orotracheal intubation. The anatomical differences explain the greater potential for technical difficulties of the airways in infants than in teenagers or adults. In general, the airways of infants differ from those of adults by five points: 1) the relatively large size of the child's tongue, in relation to the oropharynx; 2) the larynx has a more cephalic location on the neck; 3) the epiglottis is short, omegashaped and angled over the larynx entrance; 4) the vocal cords are angled, consequently, a tracheal tube that has passed blindly can easily lodge in the anterior commissure instead of sliding into the trachea; 5) the larynx is funnel-shaped, with the narrowest portion located in the cricoid cartilage. The guidelines for difficult airways in pediatrics are essential tools in this context for the prevention of associated complications, so their mastery by intensive care professionals and anesthesiologists is essential. Sharing this information is important because there is little data in the literature about approaching the airway in a patient with Mohr syndrome.
\end{abstract}

Keywords: Oro-facial-digital type II; Mohr syndrome; Airway management; Difficult airway

\section{INTRODUÇÃO}

A síndrome orofaciodigital é um transtorno raro do desenvolvimento embrionário, cuja incidência na população geral varia entre 1/1.000.000 e 1/250.000 nascidos vivos. Os pacientes podem apresentar alterações de boca, face, mãos e pés. Até o momento se desconhece a maioria dos genes implicados, sendo o diagnóstico clínico ${ }^{1}$. Há descrito pelo menos 11 formas clínicas diferentes da síndrome, entre elas a síndrome orofaciodigital tipo II ou síndrome de $\mathrm{Mohr}^{2}$. A abordagem da via aérea na síndrome orofaciodigital pode ser um desafio, já que as alterações craniofaciais como lábio 
leborino/fenda palatina, presentes na síndrome, são preditores de via aérea difícil em pediatria ${ }^{3}$. Neste artigo relatamos um caso de um paciente com diagnóstico de síndrome Mohr com foco na abordagem de via aérea difícil em pediatria.

\section{RELATO DE CASO}

Uma menina, 4 meses, peso 5,1kg, procedente de Ceilândia-DF. Apresenta lábio leporino, fenda palatina, língua e gengivas lobuladas, retrognatia e polidactilia nos quatro membros, com diagnóstico presuntivo de síndrome Orofaciodigital tipo II (Síndrome de Mohr) desde o nascimento. Adicionalmente, também tinha outras comorbidades: síndrome de Dandy Walker, forame oval patente de $2 \mathrm{~mm}$, endomiocardiofibrose, dermatite atópica e rinite alérgica. Lactente iniciou quadro de desconforto respiratório, sendo interrogada pneumonia broncoaspirativa pela médica do NRAD (Núcleo Regional de Atenção Domiciliar) e prescrito ambulatorialmente Clavulin®. No dia seguinte, sem melhora do quadro, procurou Pronto Socorro de Pediatria do HRT (Hospital Regional de Taguatinga). Foram solicitados PCR para COVID-19 e swab para B. pertussis que deram negativo. Radiografia de tórax evidenciou opacidade em hemitórax direito. Foi aventada a possibilidade de bronquiolite, sendo internada e suspendida antibioticoterapia. Nos dias subsequentes, por persistência do desconforto respiratório, foi reiniciado Clavulin ${ }^{\circledR}$ e uma nova radiografia de tórax evidenciou opacidade em terço superior direito e desvio do hilo ipsilateralmente. Após 3 dias do início dos sintomas, paciente evoluiu com piora clínica significativa, com desconforto respiratório importante e gasometria com retenção de CO2. Foi encaminhada à UTI pediátrica, onde iniciou ventilação não invasiva, com agitação extrema. Na tentativa de melhor aceitação da VNI, foi administrado 1mg de midazolam, evoluindo após com bradicardia e parada cardiorrespiratória. Protocolo de ressuscitação cardiopulmonar durou 50 minutos, recebendo 10 doses de adrenalina. 
Realizado por diversas vezes laringoscopia com lâmina reta não sendo possível visualização da glote. Após, evoluiu com gasping e retorno da circulação espontânea com hemodinâmica estável. Instalado VNI sob full face e sedoanalgesia com precedex $0,5 \mathrm{mcg} / \mathrm{kg} / \mathrm{min}$ e agendamento de traqueostomia.

\section{DISCUSSÃO}

Os dados sobre via aérea difícil pediátrica, incluindo a incidência e manejo ideal, ainda são limitados e geralmente extrapolados a partir de dados de adultos ${ }^{4}$. Dados sugerem que a incidência de laringoscopia difícil aumenta abaixo de 1 ano de idade quando comparada com bebês de mais idade $(0,24 \%-4,7 \%$ em bebês X $0,07 \%-0,7 \%$ para crianças com mais de 1 ano de idade) $)^{5}$. Apesar da incidência de via aérea difícil em pediatria ser menor, quando comparada aos adultos, as consequências de um manejo mal feito são sérias ${ }^{5}$. A maior relação ventilação alveolar/capacidade residual funcional, observada quando se comparam crianças e adultos, explica a menor reserva pulmonar, com rápida hipoxemia durante períodos relativamente curtos de hipoventilação e apneia ${ }^{6}$. Uma hipóxia precipitada pode levar rapidamente à bradicardia, assistolia e parada cardiopulmonar ${ }^{4}$.

As diferenças anatômicas explicam o potencial maior de dificuldades técnicas das vias aéreas em lactentes do que em adolescentes ou adultos. Em geral, as vias aéreas dos lactentes diferem das de adultos por cinco pontos: 1) tamanho relativamente grande da língua da criança que, em relação à orofaringe, aumenta a probabilidade de obstrução das vias aéreas e das dificuldades técnicas durante a laringoscopia; 2) a laringe tem localização mais cefálica no pescoço, tornando as lâminas retas mais úteis do que as lâminas curvas; 3 ) a epiglote é curta, em forma de ômega e angulada sobre a entrada da laringe; 4) as cordas vocais são anguladas, consequentemente, um tubo traqueal que 
passou cegamente pode facilmente se alojar na comissura anterior em vez de deslizar para dentro da traqueia; 5) a laringe é em forma de funil, sendo a porção mais estreita localizada na cartilagem cricoide ${ }^{7}$. Além do mais, a maior proeminência occipital favorece a flexão do pescoço sobre o tórax na posição supina, dificultando o alinhamento dos eixos das vias aéreas e a visualização dessas estruturas, propiciando obstrução das vias aéreas superiores ${ }^{8}$.

A síndrome orofaciodigital tipo II ou síndrome de Mohr é uma herança autossômica recessiva. Nosso paciente em questão apresentava lábio leporino, fenda palatina, língua lobulada com nódulos e polidactilia, que são alterações características da síndrome de Morh. Outras possíveis alterações são: fenótipo de baixa estatura, hipoacusia condutiva, hipertelorismo e /ou telorismo, ponte nasal baixa, ponta nasal larga ou bífida, pectus excavatum, escoliose, hipoplasia maxilar e de arco zigomático, irregularidades metafisárias, mãos com braquidactilia, hidrocefalia e/ou porencefalia ${ }^{1}$. O manejo da via aérea na síndrome orofaciodigital pode ser um desafio, já que as alterações craniofaciais como lábio leporino/fenda palatina, componentes da síndrome, são preditores de via aérea difícil em pediatria ${ }^{3}$.

Há várias limitações nos equipamentos utilizados para manejo de via aérea em crianças. Adicionalmente, há poucos guidelines de via aérea difícil em pediatria disponíveis ${ }^{9}$. A figura 1 mostra as diretrizes da Associação Pan-Indiana de Via Aérea Difíceis (AIDAA 2016) para manejo de intubação traqueal difícil não prevista em pediatria. Embora as recomendações para manejo de via aérea difícil em pediatria tenham sido em grande parte extrapoladas a partir de dados de adultos, há algumas diferenças notáveis, dentre elas: maior ênfase em manutenção constante de oxigenação (fator-chave na prevenção de rápida hipóxia e posterior bradicardia e parada cardiopulmonar); mudar para o profissional de anestesia pediátrica mais experiente após 
uma intubação fracassada com o objetivo de reduzir o potencial trauma à via aérea e posterior edema, que podem resultar em obstrução significativa em vias aéreas menores ${ }^{4}$. No relato de caso, por características específicas e limitações do sistema de saúde, não havia um profissional de anestesia pediátrica após a primeira tentativa de intubação fracassada já que se tratava de um ambiente de terapia intensiva e único plantonista na escala.

Outra diferença essencial no algoritmo da AIDAA é o último passo, onde há diferentes percursos cirúrgicos de vias aéreas determinados pela idade. Esse componente destaca a baixa taxa de sucesso de vias aéreas cirúrgicas em crianças pequenas quando tentadas por profissionais sem treinamento cirúrgico. Para pacientes com menos de 8 anos de idade, a traqueostomia cirúrgica é o procedimento preferido, já que a cricotireoidostomia pode ser de alto risco. Vale observar que intubações retrógradas em crianças são limitadas ${ }^{4}$. Uma revisão sistemática não encontrou uma clara vantagem entre cateter agulhado, bisturi ou outras técnicas cirúrgicas na via aérea de emergência pediátrica, com todas sendo associadas a altas taxas de complicações ${ }^{10}$.

É possível perceber que algumas ferramentas citadas nas diretrizes da AIDAA são de grande valia para manejo de via aérea difícil, como bougie, videolaringoscópio, estilete luminoso e broncofibroscópio. Entretanto, são instrumentos não disponíveis no serviço de saúde em questão ou, quando presentes (como videolaringoscópio e bougie), são de tamanhos inadequados para crianças. 


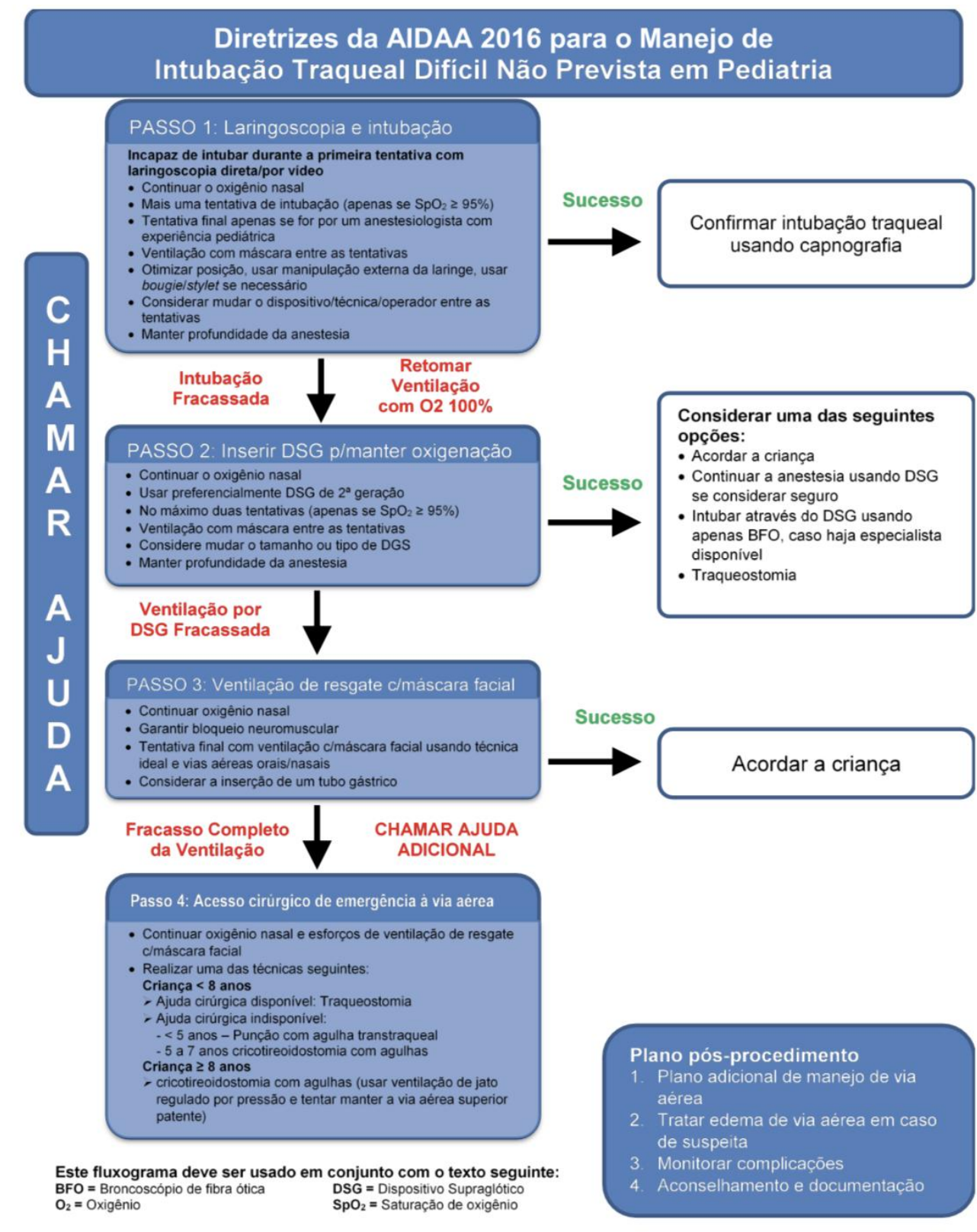

Figura 1. Diretrizes da Associação Pan-Indiana de Via Aérea Dificil para Manejo de Intubação Traqueal Dificil Não-Prevista em Pediatria (reproduzido com permissão da AIDAA). ${ }^{8}$

\section{CONCLUSÕES}

A abordagem de via aérea em pediatria pode se tornar um desafio em pacientes com doenças que envolvem alterações craniofaciais, como a Síndrome Orofaciodigital tipo II. Os guidelines de via aérea difícil em pediatria são ferramentas essenciais, nesse contexto, para prevenção de complicações associadas, sendo assim imprescindível o domínio destes por profissionais intensivistas e anestesiologistas. O compartilhamento 
dessas informações se torna importante já que há poucos dados na literatura sobre abordagem de via aérea em paciente com Síndrome de Mohr.

\section{REFERÊNCIAS}

1.Edel T, Zárate-Sanabria AG, Briceño-Balcázar I, Martínez-Lozano JC. Paciente con síndrome oro-facio-digital tipo II. Reporte del caso. Iatreia. 2017 EneMar;30(1):8691.DOI 10.17533/udea.iatreia.v30n1a09.

2.Toriello HV. Oral-facial-digital syndromes, 1992. Clin Dys- morph 1993; 2: 95-105.

3.Heinrich S, Birkholz T, Ihmsen H, Irouschek A, Ackermann A, Schimidt J. (2012). Incidence and predictors of difficult laryngoscopy in 11.219 pediatricanesthesia procedures. Pediatric Anesthesia, 22(8),729-736.

4.ATOTW 399 - Abordagem à Via Aérea Pediátrica Difícil em um Ambiente de Muitos x Poucos Recursos: Uma Comparação de Algoritmos e Carrinhos de Via Aérea Difícil (5 de março 2019).

5.Heinrich S, Birkholz $\mathrm{T}$, Ihmsen $\mathrm{H}$, et al. Incidence and predictors of difficult laryngoscopy in 11,219 pediatric anesthesia procedures. Paediatr Anaesth. 2012;22:729-736.

6. Anestesiologia: princípios e técnicas. James Manica - 4. Ed. - Porto Alegre: Artmed, 2018. Miller RD (editor). Miller`s anesthesia. $8^{\text {th }}$ ed, Philadelphia: Elsevier, 2018.

7. Miller RD (editor). Miller`s anesthesia. $8^{\text {th }}$ ed, Philadelphia: Elsevier, 2018.

8. Vialet R, Nau A. Effect of head posture on pediatric oropharyngeal structures: implications for airway management in infants and children. Curr Opin Anesthesiol, $2009 ; 23: 396-9$.

9. Walas W, Aleksandrowicz D, Borszewska-Kornacka M, Gaszynski T, Helwich E, Midgal M, Piotrowski A, Siejka G, Szczapa T, Bartkowska-Sniatkowska A. 
Unanteciipated difficult airway management in children - the consensus statement of the Paediatric Anesthesiology and Intensive Care Section and the Airway Management Section of the Polish Society of Anaesthesiology and Intensive Therapy and the Polish Society of Neonatology. Anaesthesiology Intensive Therapy 2017, vol 49, no 5, 336349.

10. Koers L, Janjatovic D, Stevens MF, Preckel B. The emergency paediatric surgical airway: a systematic review. Eur J Anaesthesiol. 2018;35(8):558-565. 